\title{
Direct observation of the effective bending moduli of a fluid membrane: Free-energy cost due to the reference-plane deformations
}

\author{
Yoshihiro Nishiyama \\ Department of Physics, Faculty of Science, \\ Okayama University, Okayama 700-8530, Japan.
}

(Dated: November 9, 2018)

\begin{abstract}
Effective bending moduli of a fluid membrane are investigated by means of the transfer-matrix method developed in our preceding paper. This method allows us to survey various statistical measures for the partition sum. The role of the statistical measures is arousing much attention, since Pinnow and Helfrich claimed that under a suitable statistical measure, that is, the local mean curvature, the fluid membranes are stiffened, rather than softened, by thermal undulations. In this paper, we propose an efficient method to observe the effective bending moduli directly: We subjected a fluid membrane to a curved reference plane, and from the free-energy cost due to the reference-plane deformations, we read off the effective bending moduli. Accepting the mean-curvature measure, we found that the effective bending rigidity gains even in the case of very flexible membrane (small bare rigidity); it has been rather controversial that for such nonperturbative regime, the analytical prediction does apply. We also incorporate the Gaussiancurvature modulus, and calculated its effective rigidity. Thereby, we found that the effective Gaussian-curvature modulus stays almost scale-invariant. All these features are contrasted with the results under the normal-displacement measure.
\end{abstract}




\section{INTRODUCTION}

Amphiphilic molecules in water segregate spontaneously into flexible extended surfaces called fluid (lipid) membranes 1, 2]. The fluid membranes are free from both surface tension and shear modulus, and the elasticity is governed only by bending rigidity [3, 4]. The Hamiltonian is given by the following form,

$$
H=\int d A\left(\frac{\kappa}{2} J^{2}+\bar{\kappa} K\right)
$$

The mean curvature $J$ is given by the summation of two principal curvatures $J=c_{1}+c_{2}$, whereas the Gaussian curvature $K$ is given by their product $K=c_{1} c_{2}$. The corresponding two moduli $\kappa$ and $\bar{\kappa}$ are called bending rigidity and Gaussian-curvature modulus, respectively. The integration $\int d A$ extends over the whole membrane surface. The Gaussiancurvature term governs the global structure of the membranes, because the term $\int d A K$ measures a topological index; for instance, for a vesicle with $n_{h}$ handles, such an identity $\int d A K=4 \pi\left(1-n_{h}\right)$ holds (Gauss-Bonnet theorem).

In spite of its seemingly simple expression, it is very hard to treat the Hamiltonian (11) by analytical methods. As a matter of fact, when written in terms of an explicit parameterization, the Hamiltonian becomes ugly; see Eqs. (??)-(??) mentioned afterwards. Hence, owing to the thermal undulations and the mutual interactions, it is expected that the effective bending moduli are modified effectively for macroscopic length scales. In order to clarify this issue, numerous renormalization-group analyses have been reported so far [5, 6, 7, 8]. For the bending rigidity, the following renormalization-group equation has been obtained;

$$
\kappa^{\prime}=\kappa-\alpha \frac{k_{B} T}{8 \pi} \ln M,
$$

with renormalized bending rigidity $\kappa^{\prime}$, temperature $T$, and the number of decimated molecules $M$. Literature agrees that the numerical prefactor in the above equation is $\alpha=3$. [A more detailed account of the historical overview would be found in Ref. 9].] Because of $\alpha>0$, the effective bending rigidity is reduced by thermally activated undulations. This conclusion might be convincing, because the membrane shape itself should be disturbed by the thermal undulations. As a matter of fact, it has been known that the orientational correlation is lost for long distances [10]. It is quite natural to anticipate that membranes become flexible for length scales exceeding this correlation length. 
Recently, however, Pinnow and Helfrich [9, 11] obtained a remarkable conclusion $\alpha=$ $-1(<0)$. The key ingredient of their new argument is that they considered the role of measure factors for the partition sum. They insist that the local mean curvature $J$ should be the right statistical measure rather than other measures such as the normal displacement $h$ and the local tilt angle $\theta$. [The normal displacement $h$ has been used as a standard measure. We will explain the $h$-based parameterization afterwards.] After an elaborated calculation of the variable replacement $h \rightarrow J$ and succeeding renormalization-group analysis, the authors reach the conclusion of $\alpha=-1$. Moreover, as for the Gaussian-curvature modulus, they insist that the effective modulus $\bar{\kappa}^{\prime}$ should remain scale-invariant;

$$
\bar{\kappa}^{\prime}=\bar{\kappa} .
$$

This conclusion again contradicts the common belief that $\bar{\kappa}$ would be enhanced for macroscopic length scales; namely, $\kappa^{\prime}=\kappa+\left(5 k_{B} T / 6 \pi\right) \ln M$ [7, 8]. This enhancement signals the topological instabilities.

The developments mentioned above all stem from the approximation that the membrane is almost flat, and the thermally excited undulations are extremely small. In our preceding paper [12], we developed an ab initio simulation scheme in order to study the thermodynamics of a fluid membrane beyond such perturbative level. As a demonstration, we calculated the transformation coefficient $d \kappa^{\prime} / d \kappa$, which yields the direction of the renormalization-group flow through coarse-graining. We found that the renormalization-group flow is influenced significantly by the choice of the statistical measures. In fact, under the mean-curvature measure, we observed an indication that the effective bending rigidity flows toward the large- $\kappa$ direction. We did not include the $\bar{\kappa}$ term in the preceding work.

In this paper, extending the preliminary analysis, we propose an efficient method to observe the effective bending moduli directly: We subject a fluid membrane to a curved reference plane. From the free-energy cost due to the reference-plane deformations, we read off the effective bending moduli. Our data indicate definitely that under the mean-curvature measure, the membrane stiffening occurs even for the case of very flexible membrane $\kappa<$ 1. This result supports the picture that the stiffening, contrary to our naive expectation, is driven by the thermal fluctuations. We also calculate the effective Gaussian-curvature modulus, and show that it remains almost scale-invariant. Again, the result is in good agreement with the Pinnow-Helfrich claim. 
It has to be mentioned that the Monte-Carlo method has been utilized successfully in the studies of membranes and vesicles [2], For the Monte-Carlo method, however, a tethered (polymerized) membrane 13] rather than a fluid membrane is more suited, because a membrane is implemented in a computer as an assembly of molecules and junctions bearing close resemblance to a tethered membrane. (Note that because of the absence of shear modulus, fluid membranes should have no internal structure.) However, Gompper and Kroll succeeded in simulating fluid membranes by the Monte-Carlo method, allowing reconstructions of junctions during the simulation [14]. They observed the topological instabilities with respect to the variation of temperature and membrane concentration. In fairness, it has to be mentioned that their Monte-Carlo data indicate softening for lipid vesicles.

The rest of this paper is organized as follows. In the next section, we describe our new approach to the direct observation of the effective bending moduli. We also explicate the outlines of the numerical method which was reported previously [12]. In Sec. ??, we present the numerical results. We are mainly concerned in the case of the mean-curvature measure. For a comparison, we will also present the data calculated under the normaldisplacement measure. With the first-principle simulation method, we will show that the scenario advocated by Pinnow and Helfrich holds true even for the non-perturbative regime. In the last section, we give summary and discussions.

\section{LEGENDRE TRANSFORMATION AND THE EFFECTIVE BENDING MOD- ULI}

In this section, we explain the idea for calculating the effective bending moduli. Mathematical formulas necessary in the succeeding numerical simulations are derived. We start with recalling the outlines of the transfer-matrix method proposed previously [12]. It is important to recognize the outlines of the transfer-matrix construction, because it elucidates the underlying physical idea of our effective-bending-moduli calculation. In short, it has to be recognized that our membrane should be classified into the "open framed membrane" in the category of Ref. [2]. 


\section{A. Transfer-matrix formalism: A brief remainder of Ref. [12]}

As mentioned in Introduction, the fluid membranes are free from shear modulus. This fact tells that the fluid membranes should have no internal structure. Hence, it is by no means fruitful to think of the microscopic constituents realizing the Hamiltonian (11). Hence, we proposed in Ref. [12], an alternative, in a sense, rather simple-minded, approach to the fluid membrane: We constructed the transfer matrix directly from the Hamiltonian (11). In the construction, we managed several discretizations which we explain below.

As noted in Introduction, the membrane shape is parameterized by the normal (transverse) displacement $h(x, y)$ from a base (reference) plane. The variables $x$ and $y$ denote the Cartesian coordinates on the reference plane. In term of this displacement field $h(x, y)$, the mean curvature and the Gaussian curvature are parameterized explicitly as follows [15];

$$
J(x, y)=\frac{\left(\partial_{x}^{2} h+\partial_{y}^{2} h\right)\left(1+\left(\partial_{x} h\right)^{2}+\left(\partial_{y} h\right)^{2}\right)-2 \partial_{x} h \partial_{y} h \partial_{x} \partial_{y} h-\partial_{x}^{2} h\left(\partial_{x} h\right)^{2}-\partial_{y}^{2} h\left(\partial_{y} h\right)^{2}}{\left(1+\left(\partial_{x} h\right)^{2}+\left(\partial_{y} h\right)^{2}\right)^{3 / 2}}
$$

and,

$$
K(x, y)=\frac{\partial_{x}^{2} h \partial_{y}^{2} h-\left(\partial_{x} \partial_{y} h\right)^{2}}{\left(1+\left(\partial_{x} h\right)^{2}+\left(\partial_{y} h\right)^{2}\right)^{2}}
$$

respectively. Similarly, the infinitesimal area $d A$ is given by,

$$
d A=\left(1+\left(\partial_{x} h\right)^{2}+\left(\partial_{y} h\right)^{2}\right)^{1 / 2} d x d y
$$

Putting them together into the Hamiltonian (11), we arrive at the explicit representation in terms of the displacement field $h(x, y)$. Now, we are led to a two-dimensional scalarfield theory with considerably complicated interactions. It is notable that the theory has even no obvious perturbation parameter. This fact motivated us to develop a first-principle simulation technique.

We put the theory on a square lattice with lattice constant $a$; see Fig. ?? (a). Accordingly, the field variables are now indexed by integer indices; namely, $h(x, y) \rightarrow h_{i j}$. Hereafter, we set the lattice constant as the unit of length; namely, $a=1$. Our theory has the translational invariance of $h \rightarrow h+\Delta h$, and thus, the absolute value of $h$ is meaningless. Therefore, it is sensible to use the link variable $\vec{s}=a \vec{\partial} h$ rather than $h$; note that $\vec{s}$ denotes the step associated at each link; see Fig. ??. We are led to the dual lattice model. Thereby, for each shaded plaquette of this dual lattice (originally a vertex spanned by four adjacent links), 
the following local statistical weight is associated;

$$
\rho\left(s_{1}, s_{2}, s_{3}, s_{4}\right)=\exp (-\mathcal{H})
$$

with,

$$
\mathcal{H}=d A\left(s_{1}, s_{2}, s_{3}, s_{4}\right)\left(\frac{\kappa}{2} J\left(s_{1}, s_{2}, s_{3}, s_{4}\right)^{2}+\bar{\kappa} K\left(s_{1}, s_{2}, s_{3}, s_{4}\right)\right),
$$

where $J\left(s_{1}, s_{2}, s_{3}, s_{4}\right), K\left(s_{1}, s_{2}, s_{3}, s_{4}\right)$ and $d A\left(s_{1}, s_{2}, s_{3}, s_{4}\right)$ are to be replaced [12] with the finite-difference versions of the differential forms (??)-(??). See Fig. ?? (b) for the definitions of $\left\{s_{\alpha}\right\}$. We have set $k_{B} T=1$, because this factor can be absorbed into the bending moduli $\kappa$ and $\bar{\kappa}$. Moreover, we should introduce yet another "statistical weight" for each open plaquette so as to impose the constraint of $\operatorname{rot} \vec{s}=0$; the gradient field should be rotationless. That is,

$$
\Delta\left(s_{1}, s_{2}, s_{3}, s_{4}\right)=\delta_{s_{1}+s_{2}-s_{3}-s_{4}, 0}
$$

To summarize, we are led to the dual lattice model [16] with the step variable $\vec{s}$. There are two types of statistical weights $\rho\left(s_{1}, s_{2}, s_{3}, s_{4}\right)(? ?)$ and $\Delta\left(s_{1}, s_{2}, s_{3}, s_{4}\right)(? ?)$, which are arranged in the checkerboard pattern. Likewise the transfer matrix is constructed as a striplike segment shown in Fig. ?? (c). It is a good position to mention a number of remarks: First, the above theory takes the displacement variable $h_{i j}$ as the statistical measure. As noted in Introduction, the mean-curvature statistical measure is considered to be physically sensible. The conversion of the statistical measure is achieved by the redefinition of the statistical weight; namely,

$$
\rho\left(s_{1}, s_{2}, s_{3}, s_{4}\right) \rightarrow \rho\left(s_{1}, s_{2}, s_{3}, s_{4}\right) \sqrt{\prod_{\alpha=1}^{4}\left|\frac{\partial J\left(s_{1}, s_{2}, s_{3}, s_{4}\right)}{\partial s_{\alpha}}\right|} .
$$

The square root is intended to take the geometrical mean, because each step variable $s_{\alpha}$ is sheared by an adjacent plaquette as well. Second, the step variable is discretized as $s_{i}=\delta_{s}\left(i-N_{s} / 2-0.5\right)$ with $i=1, \cdots, N_{s}$. The unit of step $\delta_{s}$ is determined self-consistently during the simulation by $\delta_{s}=R \sqrt{\left\langle s_{i}^{2}\right\rangle}$. This step-variable discretization is an influential factor concerning the reliability of the present simulation, and its performance was checked previously [12]. Third, in order to diagonalize the transfer matrix, we utilized the the density-matrix renormalization group [17, 18, 19, 20]. The method was invented, originally, so as to investigate the highly-correlated systems such as the Hubbard models and the spin chains. Later on, it was extended to the field of soft materials such as the lattice vibrations 
21, 22, 23], the quantum string [24, 25], and the bosonic systems [26, 27]. We repeat densitymatrix renormalization one after another so as to reach sufficiently long transfer-matrix strip length [12]. The number of states retained for a renormalized block is an important technical parameter which is denoted by $m$.

One may wonder that the strong deformations increase the area of the piece of membrane considered to such an extent that the emerging additional degrees of freedom for additional molecules cannot be ignored. According to the idea of Helfrich, however, the correct statistical measure is the local mean curvature that has noting to do with the "molecules." Therefore, our treatment is justified even for such strong deformations as long as we accept his idea. Strictly speaking, we are not dealing with such "molecules" at all; rather, we had just discretized the real space so as to form a square network, and the vertices are not to be regarded as molecules. In fact, the fluid membrane should have neither internal structure nor fixed connectivity, and it is not fruitful to think of microscopic constituents, and regard them as the degrees of freedom.

Finally, in the above, we have postulated the presence of a reference plane from which all undulations are created, and we imposed no restriction to the total area of the membrane. Hence, in terms of the category of Ref. [2], the membrane is to be classified into "open framed membrane;" see Ref. [29] as well. The free energy per unit area of the reference plane is the naturally observable quantity. This leads to the idea that the effective bending moduli would be measured from the increase of the stress energy due to the reference-plane deformations. We will pursue this idea in the next subsection.

\section{B. Legendre transformation and the effective bending moduli $\kappa_{\text {eff }}^{(S, L)}$ and $\bar{\kappa}_{e f f}^{(S, L)}$}

In this subsection, we explain our approach to the effective bending moduli. There are two types of bending moduli such as $\kappa$ and $\bar{\kappa}$; see Eq. (11). First, we explain the way to calculate the effective bending rigidity. We introduce the following Hamiltonian with an additional term;

$$
H_{C}=H-\sum_{i} C J_{i}=\sum_{i}\left(\frac{\kappa J_{i}^{2}}{2} d A_{i}+\bar{\kappa} K_{i} d A_{i}-C J_{i}\right)
$$

The index $i$ runs over the shaded plaquettes of Fig. ??; The quantities $J_{i}, K_{i}$, and $d A_{i}$ are the same as those in Eq. (??), but possessing the plaquette index $i$ now. Note that the 
additional term is not lumped together with the factor $d A_{i}$, because our aim is to calculate the stress energy with respect to the reference plane rather than the membrane surface itself. The additional term breaks the symmetry of the mean curvature $J \leftrightarrow-J$ linearly. In other words, the membrane is forced to bend so as to possess a non-vanishing spontaneous mean curvature. Hence, with respect to the stress energy due to the reference-plane deformation, we are able to observe the effective bending rigidity. That is the basic idea of our approach. Such idea was featured in an analytical treatment as well [9]. By the way, our aim is to put forward this idea to an actual computer simulation.

As is well-known, the above idea is best formulated by the Legendre transformation;

$$
G(j)=F(C)+C j,
$$

with $\partial F / \partial C=-j$. Here, $F(C)$ denotes the free energy of the Hamiltonian (??) per unit cell (one shaded plaquette). $G(j)$ is the desired Legendre-transformed free energy, which is a function of the spontaneous mean curvature $j$. Our concern is to obtain the effective bending rigidity,

$$
\kappa_{e f f}^{(S)}=\frac{\partial^{2} G}{\partial j^{2}} .
$$

With use of the well-known identity $\left(\partial^{2} G / \partial j^{2}\right)\left(\partial^{2} F / \partial C^{2}\right)=-1$, we obtain the expression for the effective bending rigidity;

$$
\kappa_{e f f}^{(S)}=-1 / \frac{\partial^{2} F}{\partial C^{2}} .
$$

Let us address a number of remarks: First, the free energy $F$ is readily accessible by the transfer-matrix calculation. Hence, the above formula is suited to our computer simulation. The remaining task is the numerical differentiation. We had adopted "Richardson's deferred approach to the limit" algorithm explicated in the textbook [28]. Second, we started from the Hamiltonian (??), which is defined on the reference plane rather than the original fluctuating membrane surface. Therefore, the effective bending rigidity corresponds to the elastic modulus with respect to the reference-plane deformation. That is precisely what we sought.

The expression of Eq. (??) may seem to be exceedingly formal. In fact, it may be unclear how the interaction of the curvature with the undulations is taken into account. It is noteworthy that the Hamiltonian contains the symmetry breaking term $C J_{i}$ and we have to evaluate the free energy in the presence of it. Just like the two-dimensional Ising model with the external field that has not yet been solved exactly, such problem with the 
symmetry breaking term is far from being trivial in itself, and generally, it contains valuable informations such as the interaction between the background curvature and the thermal undulations. In fact, Eq. (??) states that the second derivative with respect to $C$ yields the effective rigidity. That is the underlying idea behind the formal expression of Eq. (??). We stress that such an additional term is readily tractable by our simulation method, and owing to this advantage, we are able to access the effective rigidity in a quite straightforward manner.

We will introduce another effective bending rigidity: Through the coarse-graining depicted in Fig. ??, we obtain a coarse-grained lattice and the corresponding smeared curvature $\tilde{J}$ [12]. after rescaling the unit of length $\sqrt{2} a \rightarrow a$ and reexpressing the formulas in terms of the rescaled (smeared) quantities [we put ${ }^{\sim}$ for them], we obtain the effective rigidity for the coarse-grained membrane;

$$
\kappa_{e f f}^{(L)}=-1 / \frac{\partial^{2} \tilde{F}(\tilde{C})}{\partial \tilde{C}^{2}} .
$$

Note that two unit cells are renormalized into one coarse-grained unit cell. In other words, two molecules are renormalized into one decimated molecule, and hence, the parameter $M$ in Eq. (2) should be $M=2$.

Let us turn to the Gaussian-curvature modulus. In this case, we incorporate the following additional term coupling to the Gaussian curvature linearly;

$$
H_{D}=H-\sum_{i} D K_{i}=\sum_{i}\left(\frac{\kappa J_{i}^{2}}{2} d A_{i}+\bar{\kappa} K_{i} d A_{i}-D K_{i}\right) .
$$

Similar to the above, this leads to the following Legendre transformation,

$$
G(k)=F(D)+D k,
$$

with $\partial F / \partial D=-k$. Our concern is to obtain the effective Gaussian-curvature modulus, which, in other worlds, the effective symmetry breaking term with respect to the $K$ field. Postulating that the effective free energy $G(k)$ is a quadratic polynomial in terms of $k$, we obtain the following expression,

$$
\kappa_{e f f}^{(S)}=-\frac{\partial F}{\partial D} / \frac{\partial^{2} F}{\partial D^{2}}
$$

Again, the similar idea applies to the coarse-grained lattice. Hence, we obtain,

$$
\kappa_{e f f}^{(L)}=-\frac{\partial \tilde{F}}{\partial \tilde{D}} / \frac{\partial^{2} \tilde{F}}{\partial \tilde{D}^{2}} .
$$


We complete preparing the mathematical formulas for the effective bending moduli. We apply these formulas to the computer simulation in the next section.

\section{NUMERICAL CALCULATION OF THE EFFECTIVE BENDING MODULI: ROLE OF THE STATISTICAL MEASURES}

In this section, we explore the effective bending moduli $\left[\kappa_{\text {eff }}^{(S, L)}\right.$, Eqs. (??) and (??); and $\bar{\kappa}_{\text {eff }}^{(S, L)}$, Eqs. (??) and (??)] of a fluid membrane by means of the transfer-matrix method explained in Sec. ??. The transfer matrix is diagonalized [12] by means of the densitymatrix renormalization group [17, 18, 19]. Making a comparison between the results under the mean-curvature and the normal-displacement measures, we will elucidate the role of statistical measures. As mentioned in the above section, we have fixed the temperature $\left(k_{B} T=1\right)$, because this factor can be absorbed into the redefinition of the bending moduli.

Here, we shall outline some technical points, that are relevant to the simulation precision. [Detailed account of the simulation algorithm is presented in Ref. 12].] We repeated forty renormalizations for obtaining each plot; namely, the strip length of the transfer matrix extends to $L=80$. The technical parameters $m, N_{s}$ and $R$ are indicated in each figure caption; see Sec. ?? for the meanings of these technical parameters. These parameter values are equivalent or even improved to those used previously [12]. Therefore, the reliability of the simulation is maintained.

\section{A. Effective bending rigidity $\kappa_{\text {eff }}^{(S, L)}$}

In this subsection, we focus our attention on the effective bending rigidity. For that purpose, for the time being, we drop the Gaussian-curvature term, which is considered in the next subsection; namely, we set $\bar{\kappa}=0$. In Fig. ??, we plotted the effective bending rigidity $\kappa_{\text {eff }}^{(S, L)}$ for various bare rigidity $\kappa$. The moduli $\kappa_{\text {eff }}^{(S)}$ and $\kappa_{\text {eff }}^{(L)}$ denote the effective bending rigidities for smaller and longer length scales, respectively; see Eqs. (??) and (??). Here, we have accepted the local mean curvature $J$ as for the statistical measure; recent through discussion [9, 11] insists that this statistical measure should be the right one. From

the plot, we see that the rigidities $\kappa_{\text {eff }}^{(S)}$ and $\kappa_{\text {eff }}^{(L)}$ deviate from each other as for small $\kappa$. In the small- $\kappa$ regime, the membrane becomes very flexible, and so the thermal fluctuations 
should be enhanced significantly. Hence, we see that the correction to the effective rigidity is actually induced by the thermal fluctuations. Moreover, we notice $\kappa_{e f f}^{(L)}>\kappa_{\text {eff }}^{(S)}$. Hence, the membrane acquires stiffness for longer length scales; namely, the membrane stiffening sets in. Such membrane stiffening was first predicted by the analytical arguments [9, 11]. However, for the small- $\kappa$ regime, the analytical arguments are not fully justified, because the arguements stem from the "nearly flat approximation." On the other hand, our firstprinciple simulation does not rely on any perturbative treatment. In that sense, our data demonstrate very definitely that the membrane stiffening occurs withstanding the thermal disturbances.

Note that the effective rigidity $\kappa_{\text {eff }}^{(S)}$ is by no means identical to the "bare" coupling constant $\kappa$. The former is the bending elasticity with respect to the reference-plane deformations, whereas the latter is the elastic constant of the membrane surface itself. Therefore, they need not coincide. However, for sufficiently large $\kappa$, as is seen from Fig. ??, the curve tends to be parallel to the slope of the line $\kappa_{\text {eff }}^{(S)}=\kappa$, indicating that they coincide asymptotically for large $\kappa$.

We shall argue the relationship between the above result and our previous report [12]. The regime $\kappa<0.4$, where we found a notable deviation of $\kappa_{\text {eff }}^{(S)}$ and $\kappa_{\text {eff }}^{(L)}$ in Fig. ??, coincides with the area of the prominent $\partial \kappa^{\prime} / \partial \kappa$ enhancement reported in Fig. 7 of Ref. 12]. [Although $\partial \kappa^{\prime} / \partial \kappa$ does not yield direct assessment of the effective rigidity, we concluded that the $\partial \kappa^{\prime} / \partial \kappa$ enhancement should reflect the membrane stiffening.] Hence, in retrospect, our preceding analysis appears to capture the precursor of the membrane stiffening fairly correctly.

In Fig. ??, at $\kappa \approx 0.4$, there appears a singularity: The moduli $\kappa_{\text {eff }}^{(S)}$ and $\kappa_{\text {eff }}^{(L)}$ approach to each other, and for $\kappa>0.4$, they split off again. This singularity may indicate an onset of a phase transition. For $\kappa>0.4$, because of $\kappa_{\text {eff }}^{(L)}>\kappa_{\text {eff }}^{(S)}$, a membrane stiffening should occur. As a matter of fact, because of the discretization of the step variables [see Sec. ??], it is likely that the membrane is trapped by the most stable configuration (flat surface) for large $\kappa$. [It is expected that the membrane becomes flat just like the smooth phase in the solidon-solid model with large surface tension.] Moreover, it is well known that the correlation length diverges exponentially for large $\kappa[10]$. Such long correlation length would exceed the capability of the numerical simulation. The large- $\kappa$ behavior appearing in $\kappa>0.4$ is thus an artifact of the numerical simulation. The membrane stiffening for large $\kappa$ is not intrinsic, 
and is rather driven by the mechanism different from that of the small- $\kappa$ side.

In Fig. ?? (a), keeping such drawback in mind, we have drawn an anticipated behavior of the effective bending rigidity for a wide range of $\kappa$. As mentioned above, the analytical treatment is justified for large $\kappa$. On the other hand, our first-principle simulation works efficiently in the other side (non-perturbative regime). For sufficiently large $\kappa$, the analytical argument predicts the correction to the effective $\kappa$ such as $\kappa^{\prime}-\kappa=\ln M / 8 \pi=0.027 \ldots$ The correction appears to be exceedingly small to be resolved by the numerical simulation; see Fig. ?? as well. On the other hand, for the small- $\kappa$ regime, our simulation data indicate that the correction to the effective $\kappa$ increases very significantly. The amount of correction is comparable to the thermal-fluctuation energy; note that we have chosen $k_{B} T$ as the unit of energy $\left(k_{B} T=1\right)$. Hence, it is suggested that for macroscopic length scales, the membrane acquires a considerable amount of effective stiffness, and it would look almost flat irrespective of the thermal disturbances.

Let us turn to the normal-displacement statistical measure. In Fig. ??, we plotted the effective bending rigidity $\kappa_{\text {eff }}^{(S, L)}$ under this statistical measure. Notably enough, the behavior is quite contrastive with that of the local-curvature measure mentioned above: The largerscale effective rigidity $\kappa_{e f f}^{(L)}$ is suppressed by the thermal undulations. Hence, it is shown that the membrane is softened effectively for macroscopic length scales. This result may meet our intuition, and has been predicted by numerous analytical arguments based on the normal-displacement measure [5, 6, 7, 8]. We stress that our first-principle simulation covers various statistical measures in a unified way. Our simulation clarifies fairly definitely that the choice of measure factors is vital for the thermodynamics of the fluid membrane. Again, for large $\kappa \approx 0.8$, a signature of the membrane stiffening $\kappa_{\text {eff }}^{(L)}>\kappa_{\text {eff }}^{(S)}$ comes up, and the membrane should undergo the flat phase. This behavior is a drawback of our simulation as mentioned above. Keeping this in mind, we have drawn a schematic behavior of the effective bending rigidity in Fig. ?? (b). For large $\kappa$, the analytical argument predicts the renormalization correction $\kappa^{\prime}-\kappa=-3 \ln M / 8 \pi=-0.082 \ldots$, which is beyond the resolution of the present numerical simulation. As for the small- $\kappa$ regime, the correction is enhanced. However, the enhancement is not so prominent as in the case of the mean-curvature measure. It is almost comparable to the analytical prediction; see Fig. ?? as well. Hence, our firstprinciple simulation indicates that the analytical formula (2) is more or less applicable even for the case of the non-perturbative regime under the normal-displacement measure. 


\section{B. Effective Gaussian-curvature modulus $\bar{\kappa}_{\text {eff }}^{(S, L)}$}

In the above, we have studied the thermal-fluctuation-induced corrections to the bending rigidity $\kappa$. In this subsection, we incorporate the Gaussian-curvature modulus $\bar{\kappa}$, and look into its effective strength $\bar{\kappa}_{\text {eff }}^{(S, L)}$. As noted in Introduction, the Gaussian-curvature-modulus term is related to the topological index, and hence, it governs the global structure of the membranes. Roughly speaking, for $\bar{\kappa}>0$, the plumber's-nightmare phase (lamellar with tunnel-like defects) is stabilized, whereas for $\bar{\kappa}<0$, the formation of vesicles (droplets) is favored. In that sense, the quantity $\bar{\kappa}_{\text {eff }}^{(S, L)}$ reflects the tendencies toward the topological instabilities.

In Figs. ?? and ??, we presented the effective Gaussian-curvature modulus for various bare $\bar{\kappa}$ under the statistical measures of the mean curvature and the normal displacement, respectively. Here, $\kappa$ is fixed to be $\kappa=2 / \sqrt{2}(=0.35 \ldots)$ and 0.4 for respective figures. First, let us argue the latter. [Because this case provides a prototypical example, we will argue it prior to the mean-curvature case.] The latter case has been studied extensively so far with analytical approaches [7, 8]. However, because we are supposing that the membrane is framed by the reference plane, there emerge some characteristic features. For $\bar{\kappa} \approx 0$, we see that a large amount of effective $\left|\bar{\kappa}_{e f f}^{(S)}\right|$ appears. This result indicates that the membrane undulations are dominated by the dimple-like deformations, and possibly, the membrane tends to form droplets. This feature is in accordant with the previous claim [30] that for small membrane concentration, the membranes are thermodynamically unstable to the dissolution into the solvents (sponge phase). On the contrary, the effective modulus for the longer length scale, namely, $\left|\kappa_{\text {eff }}^{(L)}\right|$, exhibits considerable suppression. Notably enough, it becomes even positive for $\bar{\kappa}>0.1$ : For macroscopic length scales, the membrane recovers its stability around $\bar{\kappa} \approx 0$, although microscopic undulations are in favor of droplets. Such crossover behavior is convincing, because the membrane is framed by the reference plane, and macroscopically, lamellar-type structure should be retained.

As the bare modulus $\bar{\kappa}$ decreases, the effective moduli of different length scales coincide at $\bar{\kappa} \sim-0.6$. For the region exceeding this point $\bar{\kappa}<-0.6$, in turn, $\bar{\kappa}_{\text {eff }}^{(L)}$ dominates $\bar{\kappa}_{\text {eff }}^{(S)}$ eventually. Hence, in this region, the droplet formation is favored for macroscopic length scales. The location of this transition point is reminiscent of that advocated by the analytical arguments [31, 32], which predicts the transition point $\bar{\kappa}_{c} \approx-10 \kappa / 9=-0.44 \ldots$ In our 
simulation, the planar-type morphology is assumed a priori. Therefore, in such regime $\bar{\kappa}<\bar{\kappa}_{c}$, our numerical simulation does not cover such sponge phase. In fact, it suffers from pathologies such as the diverging mean deviation of the step variables. In the same way, for exceedingly large $\bar{\kappa}>0.1$, the membrane becomes unstable owing to the topological instability toward the plumber's-nightmare phase. In the regions depicted in Figs. ?? and ??, the planar-type morphology is retained, and thus our simulations are reliable.

Second, let us turn to the case of the mean-curvature measure: In Fig. ??, we plotted $\bar{\kappa}_{\text {eff }}^{(S, L)}$ for various bare Gaussian-curvature modulus $\bar{\kappa}$. There appear some behaviors characteristic of the mean curvature measure: We notice that $\bar{\kappa}_{\text {eff }}^{(S)}$ and $\bar{\kappa}_{\text {eff }}^{(L)}$ almost overlap each other around $\bar{\kappa} \approx 0$. That is, the Gaussian-curvature modulus stays almost scaleinvariant through coarse-graining. This result supports the aforementioned analytical prediction [9, 11] of Eq. (3). In addition to this, it is to be noted that $\bar{\kappa}_{\text {eff }}^{(S, L)}$ exhibits a large negative residual value $\bar{\kappa}_{\text {eff }}^{(S, L)} \approx-1.4$ around $\bar{\kappa} \approx 0$. That is, although the bare coupling $\bar{\kappa}$ is turned off, the membrane undulations are dominated by the dimple-like deformations. This result validates the postulation by Helfrich [9] insisting that the elementary excitations of the thermal undulations should be the "hat excitations" rather than the ordinary sinusoidal ones. Because the hat-excitation picture is the starting point of his arguments. the whole theoretical theoretical structure appears to be selfconsistent from our first-principle data.

\section{SUMMARY AND DISCUSSIONS}

We have investigated the effective bending moduli, $\kappa_{\text {eff }}^{(S, L)}$ [Eqs. (??) and (??)] and $\bar{\kappa}_{\text {eff }}^{(S, L)}$ [Eqs. (??) and (??)], with an emphasis on the role of the statistical measures for the partition sum. We employed the transfer-matrix method developed in our preceding paper [12], where we had reported a preliminary analysis on the effective bending rigidity via the transformation coefficient $\partial \kappa^{\prime} / \partial \kappa$. In the present paper, we proposed the scheme to determine the effective bending moduli directly: We calculated the free-energy cost due to the reference-plane deformations, from which we read off the effective bending moduli. This idea is formulated in terms of the Legendre transformation, and the mathematical formalism is developed in Sec. ??. Based on the formulas, we carried out extensive computer simulations in Sec. ??. As a result, we found a clear evidence of the membrane stiffening in the case of the mean-curvature measure; see Figs. ?? and ?? (a). The membrane stiffening 
was first predicted by the analytical approaches [9, 11], which are validated for sufficiently large $\kappa$. Our first-principle data show that the membrane stiffening occurs even for the nonperturbative $($ small $\kappa$ ) regime withstanding the thermal disturbances. Surprisingly enough, the enhancement of the effective bending rigidity copes with the thermal-fluctuation energy $\sim k_{B} T$, suggesting that the membrane would stay almost flat for macroscopic length scales.

On the contrary, under the normal-displacement statistical measure, we found a clear indication of the membrane softening; see Figs. ?? (b) and ??. This fact indicates that the choice of measure factors is indeed significant. The correction to the effective bending rigidity appears to be moderate compared with that of the mean-curvature measure. In fact, it is almost comparable to the prediction by the analytical treatment of Eq. (2) even for small $\kappa$.

For exceedingly large rigidity, the membrane fluctuations freeze because of the exponentially diverging correlation length [10] and the pinning potential due to the step-variable discretization; the membrane undergoes the flat phase eventually just like the solid-on-solid model with large surface tension. The appearance of such phase is a drawback of the numerical simulation, and in this respect, the simulation and the analytical treatment are both complementary.

Furthermore, we incorporated the Gaussian-curvature modulus, and studied its effective strength. Accepting the mean-curvature measure, we found that the effective Gaussiancurvature moduli for different length scales overlap each other around $\bar{\kappa} \approx 0$; see Fig. ??. In other words, the Gaussian-curvature modulus stays almost scale-invariant through coarsegraining. This fact is in good agreement with the analytical prediction of Eq. (3). In addition to this, the effective Gaussian-curvature modulus exhibits a large negative residual value even for zero bare modulus. This fact indicates that the membrane fluctuations are governed by the dimple-like deformations that should be scale-free. This observation again supports the scenario of Ref. 9] insisting that the thermal undulations should be decomposed into the hat excitations.

To conclude, by means of the first-principle simulation technique, we have investigated the series of analytical predictions advocated by Pinnow and Helfrich [9, 11]. Thereby, we found that these predictions hold true even for the non-perturbative regime. In particular, the hat-excitation picture, which is the very starting point of their argument, is validated by our $\bar{\kappa}_{e f f}^{(S, L)}$ data. Therefore, the postulation and its deductive hypotheses turn out to be 
fairly consistent as a whole. Hence, it is very likely that the mean curvature is indeed a physically sensible statistical measure for the partition sum.

\section{Acknowledgments}

This work is supported by Grant-in-Aid for Young Scientists (No. 13740240) from Monbusho, Japan.

[1] R. Lipowsky, Nature 349, 475 (1991).

[2] L. Peliti, Fluctuating geometries in statistical mechanics and field theory, edited by F. David, P. Ginsparg, and J. Zinn-Justin (Elsevier Science, Amsterdam, 1996).

[3] P. Canham, J. Theor. Biol. 26, 61 (1970).

[4] W. Helfrich, Z. Naturforsch. Teil C 28, 693 (1973).

[5] L. Peliti and S. Leibler, Phys. Rev. Lett. 54, 1690 (1985).

[6] D. Förster, Phys. Lett. A 114, 115 (1986)

[7] H. Kleinert, Phys. Lett. A 114, 263 (1986)

[8] F. David Statistical Mechanics of Membranes and Surfaces ed. D. Nelson, T. Piran and S. Weinberg (World Scientific, Singapore 1989) p. 157-223.

[9] W. Helfrich, Eur. Phys. J. B 1, 481 (1998).

[10] P. G. de Gennes and C. Taupin, J. Phys. Chem. 86, 2294 (1982).

[11] H. A. Pinnow and W. Helfrich, Eur. Phys. J. E 3, 149 (2000).

[12] Y. Nishiyama, Phys. Rev. E 66, 061907 (2002).

[13] Y. Kantor, M. Kardar and D. R. Nelson, Phys. Rev. Lett. 57, 791 (1986).

[14] G. Gompper and D. M. Kroll, J. Phys.: Condens. Matter 12, A29 (2000).

[15] P. M. Chaikin and T. C. Lubensky, Principles of Condensed Matter Physics (Cambridge University Press, 1995).

[16] Villain, J. Phys. (Paris) 36, 581 (1975).

[17] S. R. White, Phys. Rev. Lett. 69, 2863 (1992).

[18] S. R. White, Phys. Rev. B 48, 10345 (1993).

[19] T. Nishino, J. Phys. Soc. Jpn. 64, 3598 (1995). 
[20] I. Peschel, X. Wang, M. Kaulke and K. Hallberg (ed.), Density-Matrix Renormalization: A New Numerical Method in Physics (Springer-Verlag, 1999).

[21] L. G. Caron and S. Moukouri, Phys. Rev. Lett. 76, 4050 (1996).

[22] C. Zhang, E. Jeckelmann, and S. R. White, Phys. Rev. Lett. 80, 2661 (1998).

[23] A. Weiße, H. Fehske, G. Wellein, and A. R. Bishop, Phys. Rev. B 62747 (2000).

[24] Y. Nishiyama, Phys. Rev. B 64, 064510 (2001).

[25] Y. Nishiyama, Phys. Rev. B 66, 184501 (2002).

[26] Y. Nishiyama, Eur. Phys. J. B 12, 547 (1999).

[27] Y. Nishiyama, J. Phys. A: Math. Gen. 34, 11215 (2001).

[28] W. H. Press, S. A. Teukolsky, W. T. Vetterling, and B. P. Flannery, Numerical recipes in FORTRAN (Cambridge university press, 1992).

[29] J.-B. Fournier, A. Ajdari and L. Peliti, Phys. Rev. Lett. 86 (2001) 4970.

[30] G. Gompper and D. M. Kroll, Phys. Rev. Lett. 81, (1998) 2284.

[31] L. Golubović, Phys. Rev. E 50, 2419 (1994).

[32] D. C. Morse, Phys. Rev. E 50, 2423 (1994). 


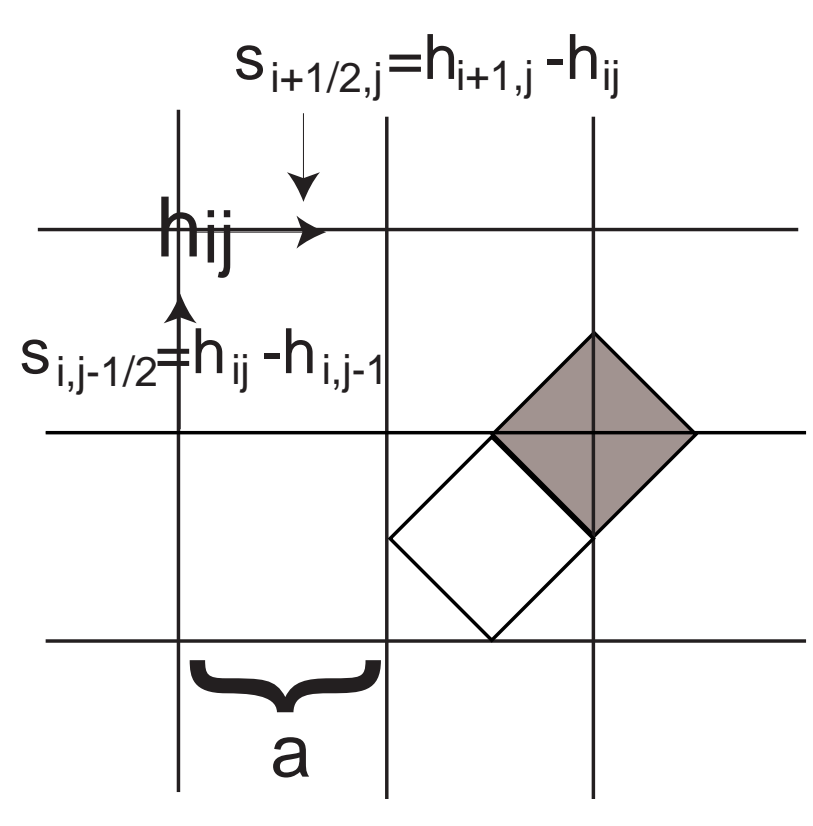

(a)

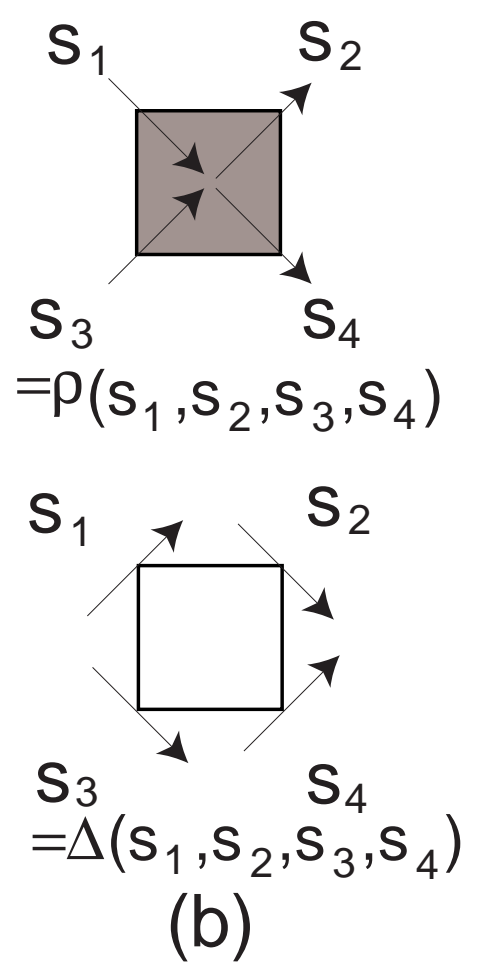

(b)

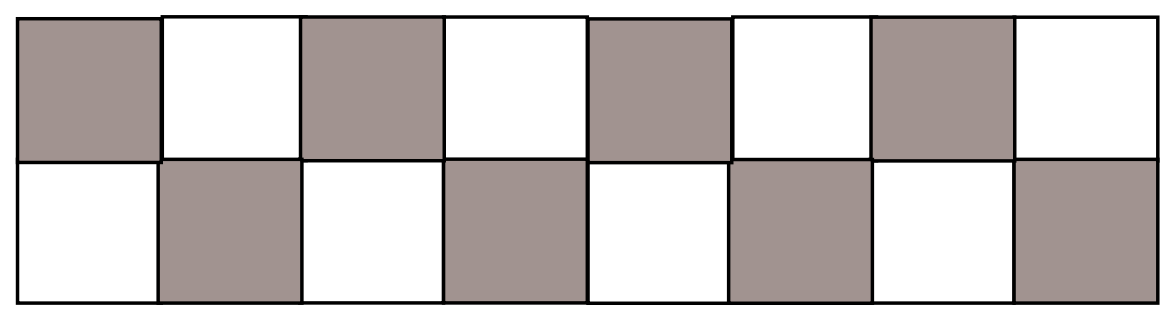

(c)

FIG. 1: (a) On the square lattice, we consider scalar field $h_{i j}$ denoting normal displacement of a membrane with respect to a reference plane. Step variable (gradient field) $\vec{s}=a \vec{\partial} h$ is defined at each link. (b) The local statistical weights $\rho$ [Eq. (??)] and $\Delta$ [Eq. (??)] are represented by shaded and open squares, respectively. The statistical weight $\rho$ has a variant so as to take account of other integration measure such as the local mean curvature (??). (c) From these local statistical weights, we construct a strip whose row-to-row statistical weight yields the transfer-matrix element. This transfer matrix is diagonalized [12] with the DMRG method [17, 18, 19]. 


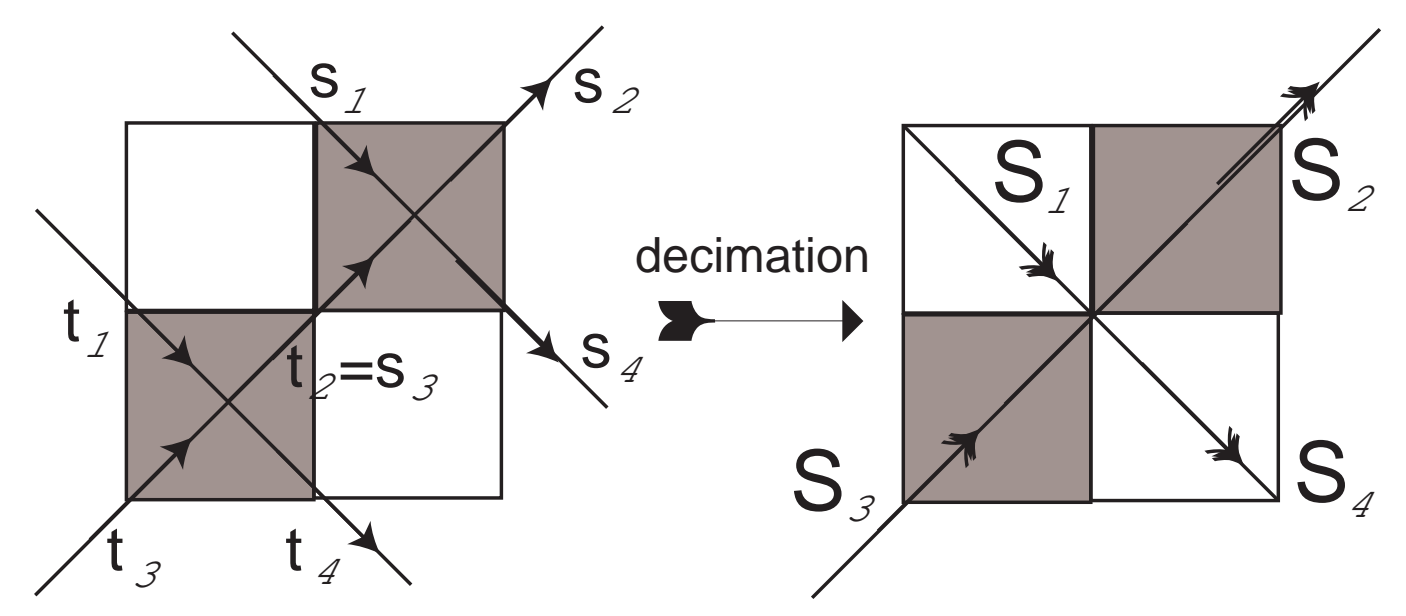

FIG. 2: Real-space decimation procedure. From the decimation, coarse-grained curvatures $\tilde{J}$ and $\tilde{K}$ are constructed [12]. $\tilde{J}$ and $\tilde{K}$ are used so as to obtain the corresponding effective bending moduli; namely, $\kappa_{\text {eff }}^{(L)}(? ?)$ and $\bar{\kappa}_{\text {eff }}^{(L)}(? ?)$. 


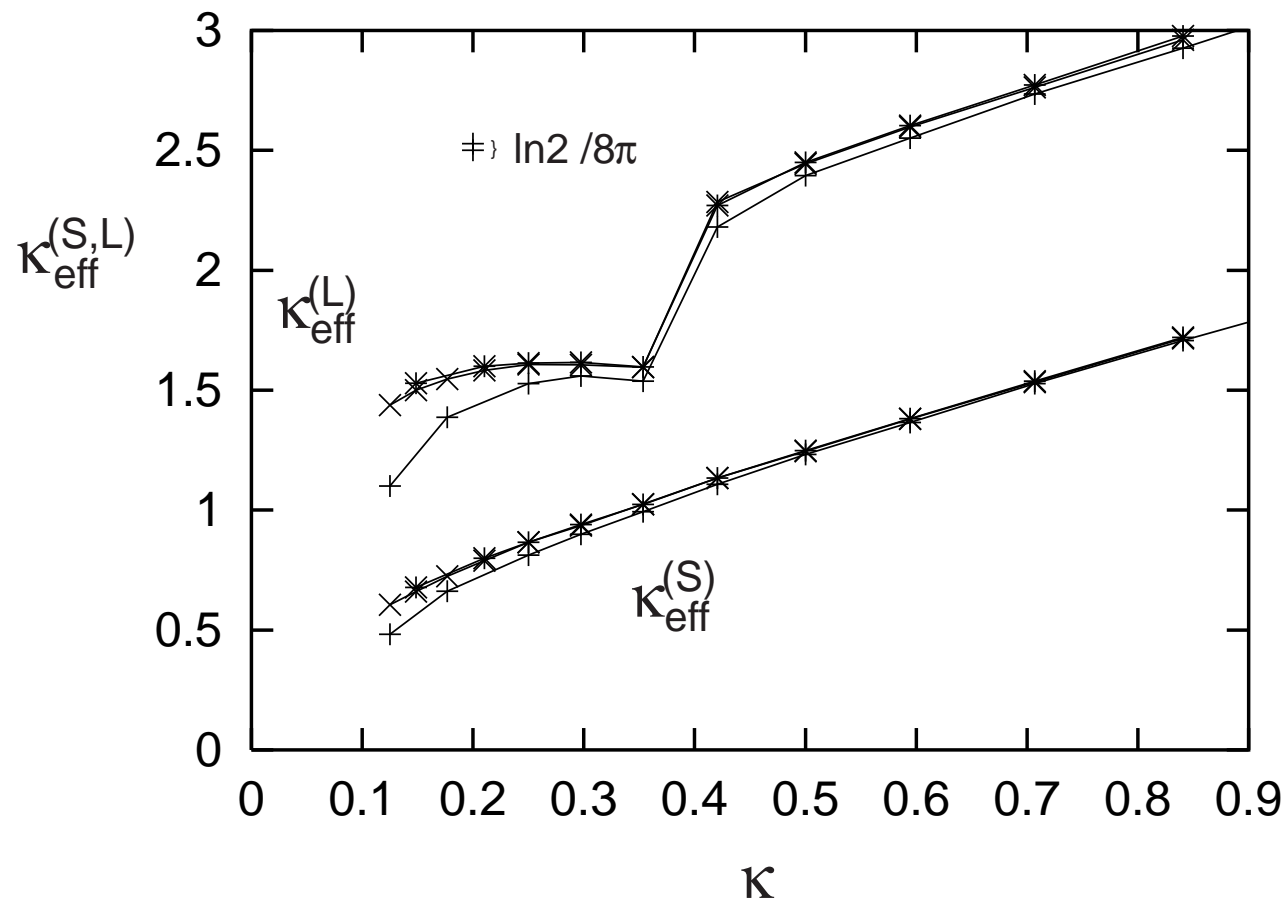

FIG. 3: Effective bending rigidity $\kappa_{\text {eff }}^{(S, L)}$ is plotted for various bare bending rigidity $\kappa$ and the fixed Gaussian-curvature rigidity $\bar{\kappa}=0$. We have accepted the local curvature as for the statistical measure. The simulation parameters for each symbol are $(+) m=15, N_{s}=8$, and $R=0.9 ;(\times)$ $m=15, N_{s}=8$, and $R=0.8$; and $(*) m=15, N_{s}=9$, and $R=0.7$. Because of $\kappa_{\text {eff }}^{(L)}>\kappa_{\text {eff }}^{(S)}$, we see that the membrane is stiffened effectively for macroscopic length scales. The stiffening in the large- $\kappa$ side, $\kappa>0.4$, may be the artifact of the numerical simulation due to the pinning potential of discretized step variables; there emerges the smooth phase just like the solid-on-solid model with large surface tension. 

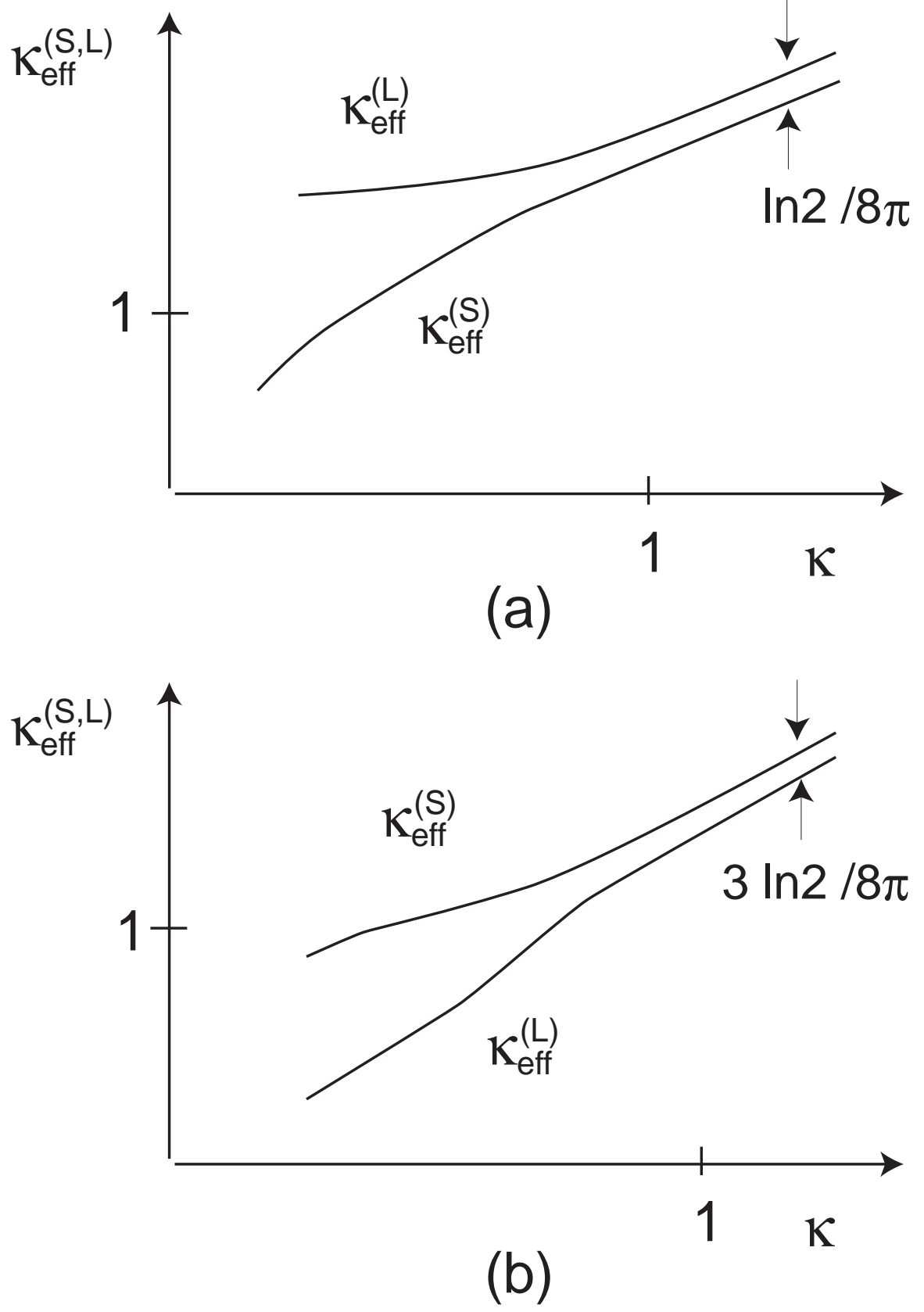

FIG. 4: Schematic drawings of $\kappa_{\text {eff }}^{(S, L)}$ anticipated from our first-principle data [Figs. ?? and ??], and the analytical result justified for sufficiently large $\kappa$ [Eq. (21)]. (a) The mean curvature is accepted as for the statistical measure. (b) The normal displacement is accepted for the statistical measure. 


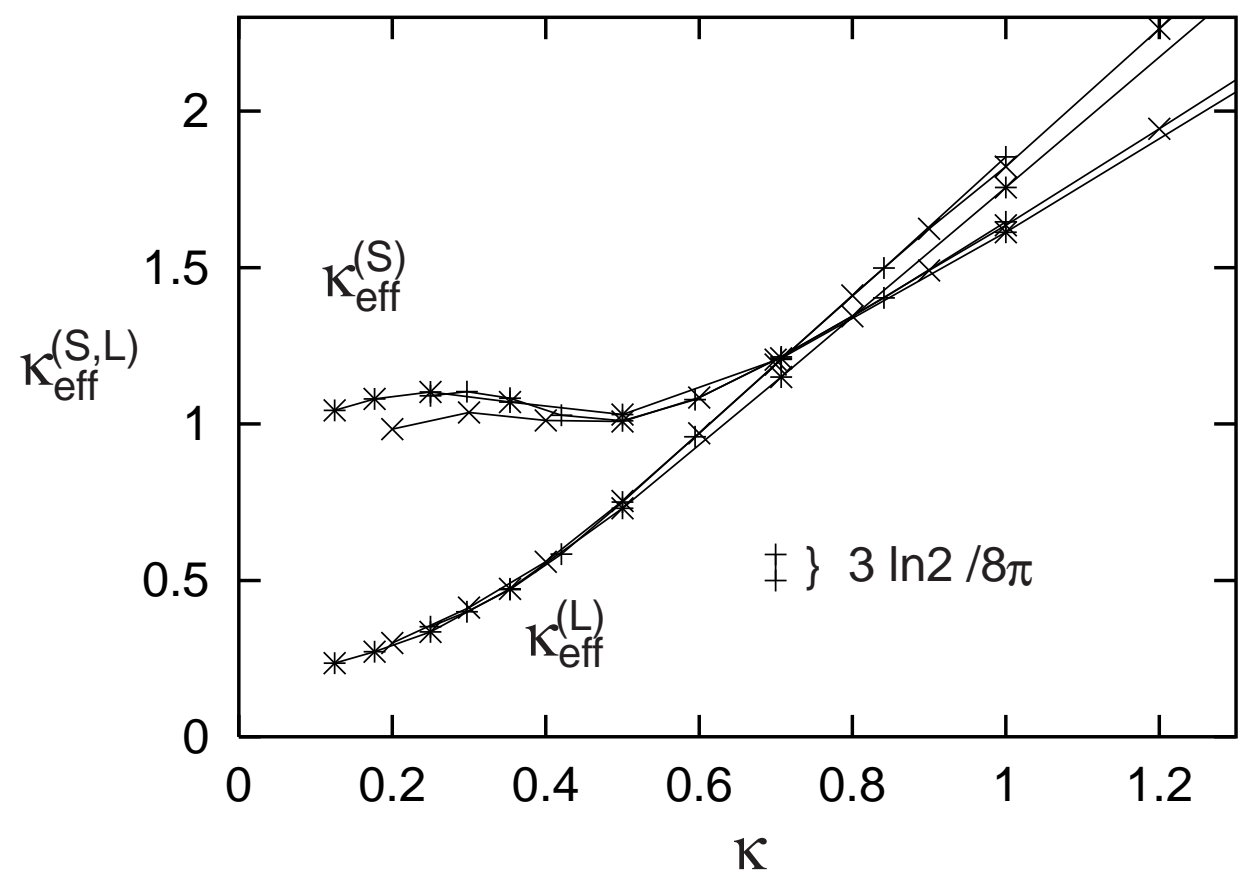

FIG. 5: Effective bending rigidity $\kappa_{\text {eff }}^{(S, L)}$ is plotted for various bare bending rigidity $\kappa$ and the fixed Gaussian-curvature rigidity $\bar{\kappa}=0$. We have accepted the normal displacement as for the statistical measure. The simulation parameters for each symbol are $(+) m=13, N_{s}=9$, and $R=0.55 ;(\times) m=10, N_{s}=11$, and $R=0.45 ;$ and $(*) m=11, N_{s}=10$, and $R=0.5$. Because of $\kappa_{\text {eff }}^{(L)}<\kappa_{\text {eff }}^{(S)}$, we see that the membrane is softened effectively for macroscopic length scales. The stiffening in the large- $\kappa$ side, $\kappa>0.8$, may be the artifact of the numerical simulation due to the pinning potential of discretized step variables; there emerges the smooth phase just like the solid-on-solid model with large surface tension. 


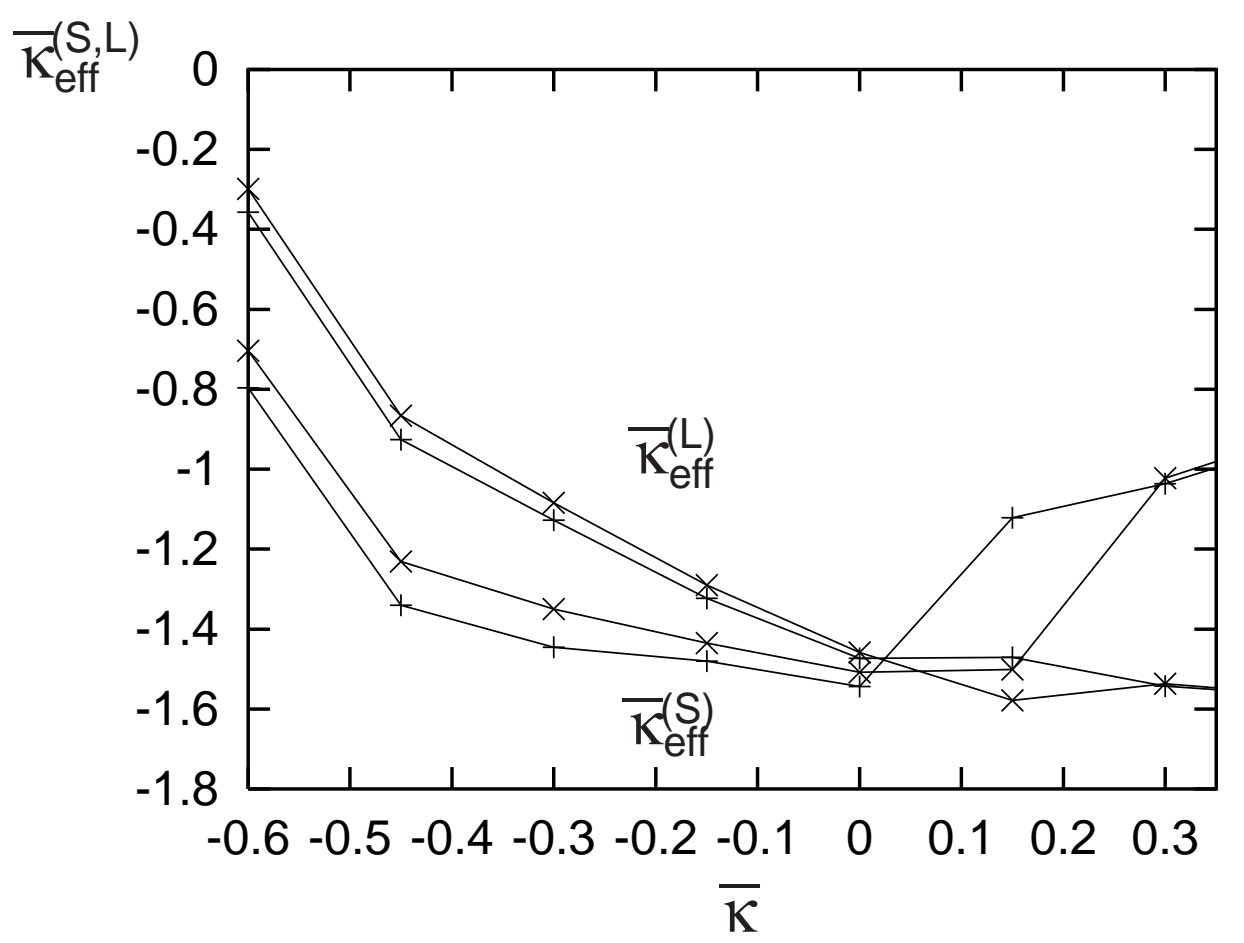

FIG. 6: Effective Gaussian-curvature modulus $\bar{\kappa}_{\text {eff }}^{(S, L)}$ [Eqs. (??) and (??)] is plotted for various bare modulus $\bar{\kappa}$ and the fixed bending rigidity $\kappa=2 / \sqrt{2}(=0.35 \ldots)$. We have accepted the local curvature as for the statistical measure. The simulation parameters for each symbol are $(+)$ $m=15, N_{s}=7$, and $R=1$; and $(\times) m=15, N_{s}=8$, and $R=0.9$. We see that the effective modulus stays almost scale-invariant around $\bar{\kappa}_{\text {eff }}^{(S, L)} \approx 0$ through coarse-graining, confirming the validity of the analytical prediction [Eq. (3)]. Moreover, we notice that $\bar{\kappa}_{\text {eff }}^{(S, L)}$ exhibits a large negative residual value even for zero bare $\bar{\kappa}=0$. This fact reflects that the membrane undulations are dominated by the formation of "hat excitations" [9]. 


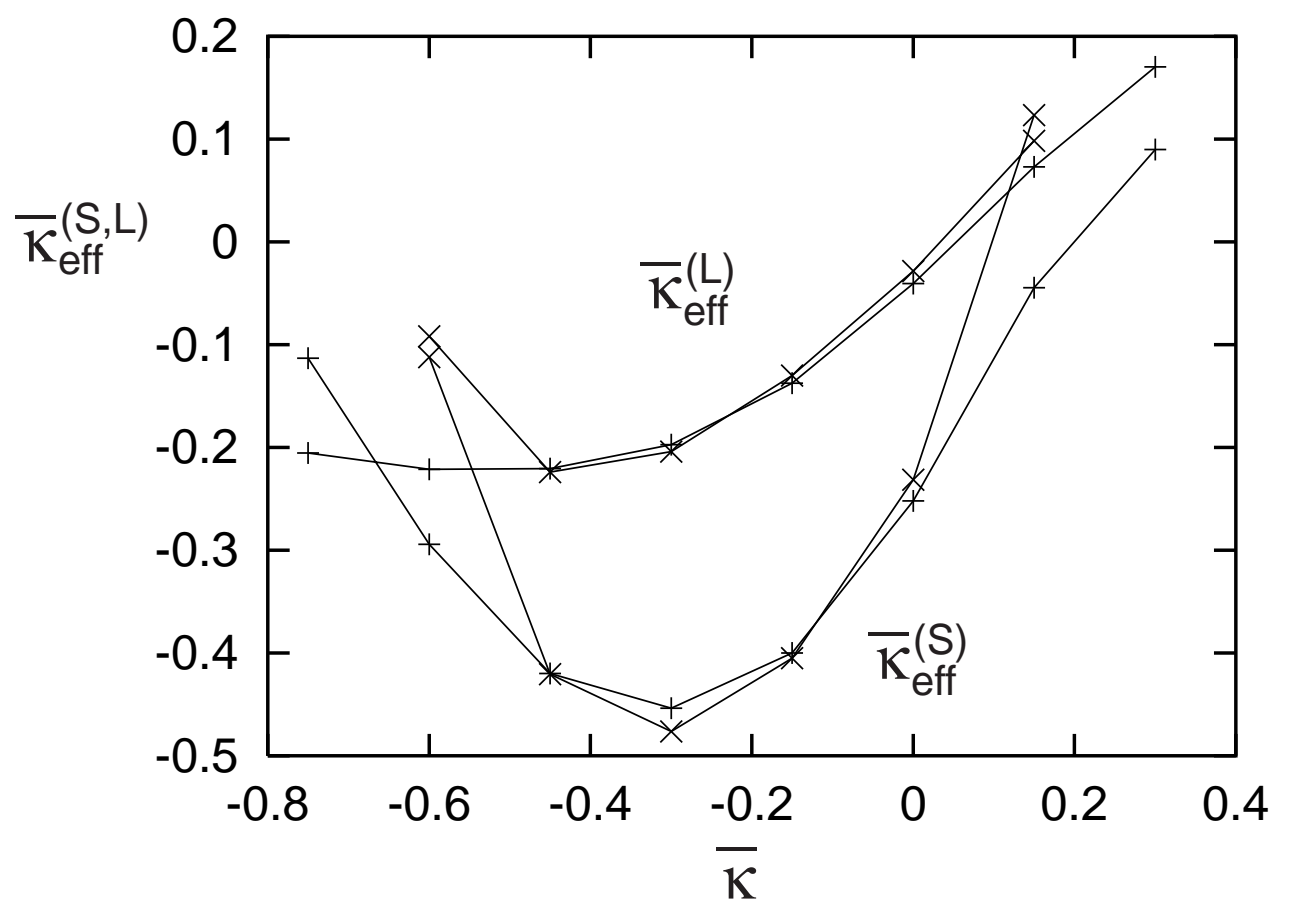

FIG. 7: Effective Gaussian-curvature modulus $\bar{\kappa}_{\text {eff }}^{(S, L)}$ [Eqs. (??) and (??)] is plotted for various bare modulus $\bar{\kappa}$ and the fixed bending rigidity $\kappa=0.4$. We have accepted the normal displacement as for the statistical measure. The simulation parameters for each symbol are $(+) m=14, N_{s}=8$, and $R=0.6$; and $(\times) m=9, N_{s}=11$, and $R=0.45$. We see that $\left|\bar{\kappa}_{\text {eff }}^{(L)}\right|$ is suppressed around $\bar{\kappa} \approx 0$. This fact tells that for macroscopic length scales (because of the restriction of the reference plane) the planar-type morphology is favored; namely, $\bar{\kappa}$ is irrelevant in the infrared limit. For $\bar{\kappa}<-0.6$, in turn, $\left|\bar{\kappa}_{\text {eff }}^{(L)}\right|$ is enhanced eventually, suggesting that the membrane tends to dissolve into the solvent (droplet phase). 